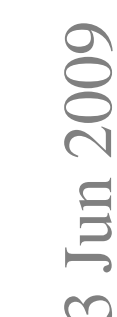

. N

$\checkmark$

U

龶

。

E

n:

$>$

8

N

$: 5$

$\vartheta$

- E

:

光 


\title{
q-Classical orthogonal polynomials: A general difference calculus approach
}

\author{
R. S. Costas-Santos (rscosa@gmail.com) \\ Department of Mathematics. University of California, Santa Barbara, CA 93106, USA. \\ F. Marcellán (pacomarc@ing.uc3m.es) \\ Departamento de Matemáticas. Universidad Carlos III de Madrid. Avenida Universidad 30, 28911-Leganés, \\ Spain
}

July 6, 2018

\begin{abstract}
It is well known that the classical families of orthogonal polynomials are characterized as the polynomial eigenfunctions of a second order homogenous linear differential/difference hypergeometric operator with polynomial coefficients.

In this paper we present a study of the classical orthogonal polynomials sequences, in short classical OPS, in a more general framework by using the differential (or difference) calculus and Operator Theory.

The Hahn's Theorem and a characterization Theorem for the $q$-polynomials which belongs to the $q$-Askey and Hahn tableaux are proved. Finally, we illustrate our results applying them to some known families of orthogonal $q$-polynomials.
\end{abstract}

Keywords: Classical orthogonal polynomials; Discrete orthogonal polynomials; $q$-Polynomials; Characterization Theorems; The Rodrigues operator

2000 MSC codes: 33C45; 33D45

\section{Introduction}

Classical orthogonal polynomials constitute a very important and interesting family of special functions. They are mathematical objects which have attracted attention not only because of their mathematical value but also because of their connections with physical problems. In fact, they are also related, among others, to continued fractions, Eulerian series, elliptic functions $[7,13]$, and quantum algebras $[17,18,28]$.

They also satisfy a three-term recurrence relation (TTRR) [24]

$$
x(s) p_{n}(x(s))=\alpha_{n} p_{n+1}(x(s))+\beta_{n} p_{n}(x(s))+\gamma_{n} p_{n-1}(x(s)), \quad n \geq 0,
$$

where $\gamma_{n} \neq 0, n \geq 1$, as well as their derivatives (or differences or $q$-differences) also constitute a a sequence of orthogonal family (see e.g. $[3,4,11,24]$ for a more recent review).

Indeed, a fundamental role is played by the so-called characterization Theorems, i.e. the Theorems which collect those properties that completely define and characterize the classical orthogonal polynomials.

One of the many ways to characterize a family $\left(p_{n}\right)$ of classical polynomials (Hermite, Laguerre, Jacobi, and Bessel), which was first posed by R. Askey and proved by W. A. Al-Salam and T. S. Chihara [1] (see also [21]), is the structure relation

$$
\phi(x) p_{n}^{\prime}(x)=\tilde{a}_{n} p_{n+1}(x)+\tilde{b}_{n} p_{n}(x)+\tilde{c}_{n} p_{n-1}(x), \quad n \geq 0,
$$

where $\phi$ is a fixed polynomial of degree at most 2 and $\tilde{c}_{n} \neq 0, n \geq 1$.

A. G. Garcia, F. Marcellán, and L. Salto [14] proved that the relation (1) also characterizes the discrete classical orthogonal polynomials (Hahn, Krawtchouk, Meixner, and Charlier polynomials) when the derivative is replaced by the forward difference operator $\Delta$ defined as

$$
\Delta f(x):=\left(\mathscr{E}^{+}-\mathscr{I}\right) f(x)=f(x+1)-f(x),
$$

where $\mathscr{E}^{+}$is the forward shift operator and $\mathscr{I}$ the identity operator. 
Later on, J. C. Medem, R. Álvarez-Nodarse, and F. Marcellán [22] (see also [2, 4, 5]) characterized the orthogonal polynomials which belong to the $q$-Hahn class by a structure relation obtained from (1) replacing the derivative by the $q$-difference operator $\mathscr{D}_{q}$ defined as

$$
\mathscr{D}_{q}(f)(x):=\frac{f(q x)-f(x)}{(q-1) x}, \quad q \in \mathbb{C},|q| \neq 0,1 .
$$

The aim of the present paper is to go further in the study of classical polynomials giving two new structure relations for the $q$-polynomials which belong to the $q$-Askey tableau and the lattice is $q$-quadratic, i.e. $x(s)=c_{1} q^{s}+c_{2} q^{-s}+c_{3}$, with $c_{1} c_{2} \neq 0$, being $q \in \widetilde{\mathbb{C}}:=\mathbb{C} \backslash$ $\left(\{0\} \cup\left(\cup_{n \geq 1}\left\{z \in \mathbb{C}: z^{n}=1\right\}\right)\right)$.

In fact, we prove that the following relation characterizes the $q$-polynomials.

$$
\mathscr{M} p_{n}(x(s))=e_{n} \frac{\Delta p_{n+1}(s)}{\Delta x(s)}+f_{n} \frac{\Delta p_{n}(s)}{\Delta x(s)}+g_{n} \frac{\Delta p_{n-1}(s)}{\Delta x(s)}, \quad n \geq 0,
$$

where $\mathscr{M}$ is the forward arithmetic mean operator:

$$
\mathscr{M}:=\frac{1}{2}\left(\mathscr{E}^{+}+\mathscr{I}\right)
$$

and, $\left(e_{n}\right),\left(f_{n}\right)$, and $\left(g_{n}\right)$ are sequences of complex numbers such that $e_{n} \neq 0$ and $g_{n} \neq \gamma_{n}$.

Furthermore, we give a characterization Theorem for classical orthogonal polynomials in a more general framework using Operator Theory, as well as we deduce some unified expressions for the second order homogeneous linear differential (or difference) hypergeometric operator and for its polynomial eigenfunctions.

The structure of this paper is as follows: In Section 2 we introduce some notations and definitions used throughout the paper. In Section 3 we define the Rodrigues Operator and using Operator Theory we deduce a unified expression for the linear differential (difference resp.) hypergeometric operators related to the classical families, and for their polynomial eigenfunctions, as well as other algebraic properties. In Section 4 we present a new characterization Theorem for $q$-classical orthogonal polynomials and we prove the Hahn's Theorem for the $q$-polynomials of the $q$-Askey tableau. In Section 5 we illustrate our results applying them to some known $q$-polynomials.

\section{Preliminaries}

The standard classical orthogonal polynomials (Hermite, Laguerre, Jacobi, and Bessel) are eigenfunctions of a second order linear differential operator [26]

$$
\mathscr{H}:=\tilde{\sigma}(x) \frac{d^{2}}{d x^{2}}+\tilde{\tau}(x) \frac{d}{d x},
$$

where $\tilde{\sigma}$ and $\tilde{\tau}$ are polynomials of degree at most 2 and 1 , respectively. This operator is also said to be a standard Hamiltonian operator (see [24]).

In fact, a sequence of monic polynomials, $\left(p_{n}\right)$, such that

$$
\mathscr{H} p_{n}(x)=\left(n \tilde{\tau}^{\prime}+n(n-1) \frac{\tilde{\sigma}^{\prime \prime}}{2}\right) p_{n}(x), \quad n \geq 0
$$

is orthogonal with respect to a certain weight function $\rho(x)$ supported on $\Omega \subseteq \mathbb{R}$, i.e.

$$
\int_{\Omega} p_{n}(x) p_{m}(x) \rho(x) d x=d_{n}^{2} \delta_{n, m}, \quad n, m \geq 0 .
$$

Notice that $\Omega$ is an interval associated with the regular singularities of $\mathscr{H}$. 
Moreover, this weight function $\rho(x)$ fulfills the following Pearson equation

$$
\frac{d}{d x}(\tilde{\sigma}(x) \rho(x))=\tilde{\tau}(x) \rho(x)
$$

On the other hand, we can consider two different discretizations of the operator (2) replacing the derivative by certain approximations on a lattice. Thus we need first to introduce the concept of lattice.

DEFINITION 2.1. [11, 24] $A$ lattice is a complex function $x \in C^{2}(\Lambda)$ where $\Lambda$ is a complex domain, $\mathbb{N}_{0} \subseteq \Lambda$, and $x(s), s=0,1, \ldots$ are the points where we will discretize (2).

For a first approximation of the operator $\mathscr{H}$, we consider the uniform lattice $x(s)=s$. Thus the following second order homogeneous linear difference hypergeometric operator

$$
\mathscr{H}_{\Delta}:=\sigma(s) \Delta \nabla+\tau(s) \Delta,
$$

can be introduced where $\nabla$ and $\Delta$ are the backward and forward difference operators, respectively, being

$$
\nabla f(x)=\left(\mathscr{E}^{-}-\mathscr{I}\right) f(x)=f(x)-f(x-1),
$$

$\sigma(x):=\tilde{\sigma}(x)-\frac{1}{2} \tilde{\tau}(x)$ is a polynomial of degree at most 2 , and $\tau(x)=\tilde{\tau}(x)$.

Indeed, a sequence of polynomials $\left(p_{n}\right)$ satisfying

$$
\mathscr{H}_{\Delta} p_{n}(x)=\left(n \tilde{\tau}^{\prime}+n(n-1) \frac{\tilde{\sigma}^{\prime \prime}}{2}\right) p_{n}(x), \quad n \geq 0,
$$

is orthogonal with respect to a certain weight function $\rho(x)$ supported on $\Omega \subseteq \mathbb{R}$, i.e.

$$
\sum_{x \in \Omega} p_{n}(x) p_{m}(x) \rho(x)=d_{n}^{2} \delta_{n, m}, \quad n, m \geq 0,
$$

with some extra boundary conditions (see $\S 2.3$ in page 26 of [24]).

As in the continuous case, the weight function $\rho$ fulfills the following $\Delta$-Pearson equation

$$
\Delta(\sigma(x) \rho(x))=\tau(x) \rho(x) .
$$

The second way to discretize the operator $\mathscr{H}$ is to consider a nonuniform lattice $x(s)$, (see [24]) which leads to the following linear difference hypergeometric operator:

$$
\mathscr{H}_{q}:=\sigma(s) \frac{\Delta}{\nabla x_{1}(s)} \frac{\nabla}{\nabla x(s)}+\tau(s) \frac{\Delta}{\Delta x(s)},
$$

where $x(s)=c_{1} q^{s}+c_{2} q^{-s}+c_{3}, q \in \widetilde{\mathbb{C}}, \sigma(s):=\tilde{\sigma}(x(s))-\frac{1}{2} \tau(x(s)) \nabla x_{1}(s), x_{1}(s):=x\left(s+\frac{1}{2}\right)$, and $\tau(s)=\tilde{\tau}(x(s))$.

Notice that, in general, $\sigma(s)$ is not a polynomial on $x(s)$ but if either $c_{1}=0$ or $c_{2}=0$ then $\sigma(s)$ is a polynomial on $x(s)$ of degree at most 2, i.e. $\sigma(s) \equiv \sigma(x(s))$.

Indeed, a sequence of monic polynomials $\left(p_{n}\right)$ satisfying

$$
\mathscr{H}_{q} p_{n}(x(s))=[n]_{q}\left(\alpha_{q}(n-1) \tilde{\tau}^{\prime}+[n-1]_{q} \frac{\tilde{\sigma}^{\prime \prime}}{2}\right) p_{n}(x(s)), \quad n \geq 0,
$$

where $[s]_{q}$ denotes the $q$-number (in its symmetric form)

$$
[s]_{q}:=\frac{q^{\frac{s}{2}}-q^{-\frac{s}{2}}}{q^{\frac{1}{2}}-q^{-\frac{1}{2}}}, \quad s \in \mathbb{C},
$$

and

$$
\alpha_{q}(s):=\frac{q^{\frac{s}{2}}+q^{-\frac{s}{2}}}{2}, \quad s \in \mathbb{C}
$$

is orthogonal with respect to a certain linear functional $\mathbf{u} \in \mathbb{P}^{\prime}$, i.e.

$$
\left\langle\mathbf{u}, p_{n} p_{m}\right\rangle=d_{n}^{2} \delta_{m, n}, \quad m, n \geq 0 .
$$


REMARK 2.1. Notice that $\mathbf{u}$ admits a Lebesgue-Stieltjes integral representation, not necessarily unique,

$$
\langle\mathbf{u}, p\rangle=\int_{\Gamma} p(z) d \mu(z),
$$

where $\Gamma$ is a contour in the complex plane and $\mu$ is a measure, with $\operatorname{supp}(\mu)=\Gamma$.

In fact, in most of the cases, such an integral can be transformed into a sum in such a way the linear functional can be written as [16]

$$
\langle\mathbf{u}, p\rangle=\sum_{s_{i} \in \Omega} p\left(x\left(s_{i}\right)\right) \rho\left(s_{i}\right) \nabla x_{1}\left(s_{i}\right), \quad s_{i+1}=s_{i}+1,
$$

where the function $\rho$ is supported on $\Omega \subseteq \mathbb{R}$. For this reason we will assume the above representation for the functional although the proofs when $\mathbf{u}$ can not be represented in such a way are analogous and we will omit them.

Moreover, as in the discrete case, the weight function $\rho(s)$ satisfies the following $q$-Pearson equation

$$
\frac{\Delta(\sigma(s) \rho(s))}{\nabla x_{1}(s)}=\tau(s) \rho(s) .
$$

REMARK 2.2. It is important to point out that in the orthogonality conditions (3), (5), and (7) one needs to impose some boundary conditions, which follow from the fact that the moments of the corresponding measure must be finite (see, for instance, [12], [24, p. 27, p. 70], [25]).

An important fact related to these operators is its hypergeometric character, i.e. if $y$ is an eigenfunction of the linear operator $\mathscr{H}, \mathscr{H}_{\Delta}$, or $\mathscr{H}_{q}$ respectively, its derivative $\tilde{y}=y^{\prime}$, its difference $\tilde{y}=\Delta y$, or its $q$-difference $\tilde{y}=\Delta^{(1)} y$, respectively, is an eigenfunction of the second order linear operator of the same kind. For instance, $\tilde{y}=\Delta^{(1)} y$ is an eigenfunction of the following second order linear difference operator:

$$
\widetilde{\mathscr{H}_{q}}:=\sigma(s) \frac{\Delta}{\nabla x_{1}(s)} \frac{\nabla}{\nabla x(s)}+\left(\tau(s+1) \frac{\Delta x_{1}(s)}{\Delta x(s)}+\frac{\sigma(s+1)-\sigma(s)}{\Delta x(s)}\right) \frac{\Delta}{\Delta x(s)},
$$

where

$$
\Delta^{(m)}:=\frac{\Delta}{\Delta x_{m-1}(s)} \frac{\Delta}{\Delta x_{m-2}(s)} \cdots \frac{\Delta}{\Delta x(s)}, \quad m \geq 1, \quad x_{m}(s):=x\left(s+\frac{m}{2}\right), \quad m \in \mathbb{Z} .
$$

Throughout the paper we will study some properties related to the difference operator $\mathscr{H}_{q}$ and its eigenfunctions.

We can rewrite $\mathscr{H}_{q}$ as follows

$$
\mathscr{H}_{q}=\frac{1}{\nabla x_{1}(s)}\left(\left(\sigma(s)+\tau(s) \nabla x_{1}(s)\right) \frac{\Delta}{\Delta x(s)}-\sigma(s) \frac{\nabla}{\nabla x(s)}\right) .
$$

Moreover, with this definition we can also rewrite the $q$-Pearson equation (8) as

$$
\sigma(s+1) \rho(s+1)=\left(\sigma(s)+\tau(s) \nabla x_{1}(s)\right) \rho(s) .
$$

Combining (9) and (10), we get the symmetric or self-adjoint form of (6)

$$
\mathscr{H}_{q}=\left[\frac{1}{\rho(s)} \frac{\nabla}{\nabla x_{1}(s)} \rho_{1}(s)\right] \circ \frac{\Delta}{\Delta x(s)},
$$

where $\rho_{0}(s):=\rho(s)$ and $\rho_{1}(s):=\rho(s+1) \sigma(s+1)$.

This representation for the difference operator (6) is the key to define the Rodrigues Operator which we will use in order to unify all the representations in the context of the classical orthogonal polynomials. 
REMARK 2.3. A direct calculation yields the analogous formulas for the operators $\mathscr{H}$ and $\mathscr{H}_{\Delta}$.

$$
\mathscr{H}=\left[\frac{1}{\rho(x)} \frac{d}{d x} \rho_{1}(x)\right] \circ \frac{d}{d x},
$$

where $\rho_{0}(x):=\rho(x)$ and $\rho_{1}(x):=\rho(x) \sigma(x)$; and

$$
\mathscr{H}_{\Delta}=\left[\frac{1}{\rho(s)} \nabla \rho_{1}(s)\right] \circ \Delta,
$$

where $\rho_{0}(s):=\rho(s)$ and $\rho_{1}(s):=\rho(s+1) \sigma(s+1)$.

Using this information we can write the polynomial eigenfunctions of the operator $\mathscr{H}_{q}$ as (see $[24,(3.2 .10)$, p. 66])

$$
p_{n}(x(s)):=\left[\frac{B_{n}}{\rho_{0}(s)} \frac{\nabla}{\nabla x_{1}(s)} \rho_{1}(s)\right] \circ\left[\frac{1}{\rho_{1}(s)} \frac{\nabla}{\nabla x_{2}(s)} \rho_{2}(s)\right] \circ \cdots \circ\left[\frac{1}{\rho_{n-1}(s)} \frac{\nabla}{\nabla x_{n}(s)} \rho_{n}(s)\right],
$$

or, after some simplifications, as

$$
p_{n}(x(s))=\frac{B_{n}}{\rho_{0}(s)} \nabla_{0}^{(n)}\left[\rho_{n}(s)\right], \quad B_{n} \neq 0,
$$

where $\rho_{0}(s)=\rho(s), \rho_{k}(s)=\rho_{k-1}(s+1) \sigma(s+1)$, for every $k \in \mathbb{Z}$, and

$$
\nabla^{(n)} \equiv \nabla_{0}^{(n)}, \quad \nabla_{k}^{(n)}:=\frac{\nabla}{\nabla x_{k+1}(s)} \frac{\nabla}{\nabla x_{k+1}(s)} \cdots \frac{\nabla}{\nabla x_{n}(s)}, \quad k=0,1, \ldots, n-1 .
$$

\subsection{The (FORWARD) MEAN ARITHMEtic PROCESS}

It is well known that for any pair of polynomials, $\pi$ and $\xi$, the following relations hold:

$$
\begin{aligned}
& \Delta^{(1)}(\pi \xi)=\left(\Delta^{(1)} \pi\right) \xi+\left(\mathscr{E}^{+} \pi\right) \Delta^{(1)} \xi, \\
& \Delta^{(1)}(\pi \xi)=\left(\Delta^{(1)} \xi\right) \pi+\left(\mathscr{E}^{+} \xi\right) \Delta^{(1)} \pi .
\end{aligned}
$$

We will say that we apply the (forward) mean arithmetic process to $\Delta^{(1)}(\pi \xi)$ if we get the arithmetic mean of the above two expressions obtaining in such a case

$$
\Delta^{(1)}(\pi \xi)=\left(\Delta^{(1)} \pi\right) \mathscr{M} \xi+\left(\Delta^{(1)} \xi\right) \mathscr{M} \pi .
$$

For example,

$$
\Delta^{(1)}\left(x(s) p_{n}(x(s))\right)=\mathscr{M} p_{n}(x(s))+\alpha_{q}(1) x_{1}(s) \Delta^{(1)} p_{n}(x(s))+c_{3}(q)\left(1-\alpha_{q}(1)\right) .
$$

In an analogous way we define the (backward) mean arithmetic mean process although we will not use it.

\section{The Rodrigues Operator}

The expressions given in the previous section for the difference operator $\mathscr{H}_{q}$ and for its polynomial eigenfunctions (12) suggest us to consider a new operator which we call the Rodrigues Operator.

DEFINITION 3.1. Given functions $\sigma$ and $\rho$, where $\rho$ is supported on $\Omega$, and a lattice $x(s)$, we define the $k$-th Rodrigues Operator associated with $\left(\sigma(s), \rho_{m}(s), x_{m}(s)\right)$ as

$$
\begin{gathered}
\mathscr{R}_{0}\left(\sigma(s), \rho_{m}(s), x_{m}(s)\right):=\mathscr{I}, \quad \mathscr{R}_{1}\left(\sigma(s), \rho_{m}(s), x_{m}(s)\right):=\frac{1}{\rho_{m}(s)} \frac{\nabla}{\nabla x_{m+1}(s)} \rho_{m+1}(s) \circ \mathscr{I}, \\
\mathscr{R}_{k}\left(\sigma(s), \rho_{m}(s), x_{m}(s)\right):=\mathscr{R}_{1}\left(\sigma(s), \rho_{m}(s), x_{m}(s)\right) \circ \mathscr{R}_{k-1}\left(\sigma(s), \rho_{m+1}(s), x_{m+1}(s)\right), k \geq 2 .
\end{gathered}
$$


For the sake of simplicity, we denote

$$
\mathscr{R}_{k}\left(\rho_{m}(s), x_{m}(s)\right):=\mathscr{R}_{k}\left(\sigma(s), \rho_{m}(s), x_{m}(s)\right), \quad m \in \mathbb{Z}, k \geq 0 .
$$

Notice that in the continuous case

$$
\mathscr{R}_{1}\left(\rho_{m}(x), x\right)=\frac{1}{\rho_{m}(x)} \frac{d}{d x} \rho_{m+1}(x) \circ \mathscr{I},
$$

and in the discrete case

$$
\mathscr{R}_{1}\left(\rho_{m}(s), x(s)\right)=\frac{1}{\rho_{m}(s)} \nabla \rho_{m+1}(s) \circ \mathscr{I} .
$$

From this definition the difference operator (11) can be written as

$$
\mathscr{H}_{q}=\mathscr{R}_{1}(\rho(s), x(s)) \circ \Delta^{(1)},
$$

and (12) reads

$$
p_{n}(x(s))=B_{n} \mathscr{R}_{n}(\rho(s), x(s))(1), \quad B_{n} \neq 0, n \geq 0 .
$$

Therefore, this is the way to write the operators $\mathscr{H}\left(\mathscr{H}_{\Delta}\right.$ or $\mathscr{H}_{q}$, resp.) and their corresponding polynomial eigenfunctions in a unified way.

Now, let us consider other properties related to the Rodrigues Operator.

LEMMA 3.1. The Pearson-type equation (8) can be written as follows

$$
\mathscr{R}_{1}(\sigma(s), \rho(s), x(s))(1)=\tau(s) .
$$

As a consequence we get result:

LEMMA 3.2. [24, (3.2.18), p.66] For every integers $n, k, 0 \leq k \leq n$, there exists a constant, $C_{n, k}$, such that

$$
\Delta^{(k)} p_{n}(x(s))=C_{n, k} \mathscr{R}_{n-k}\left(\rho_{k}(s), x_{k}(s)\right)(1) .
$$

\section{The characterization Theorem for the $q$-classical polynomials}

The characterization Theorems constitute a very useful tool to analyze classical orthogonal polynomials as we stated in the introduction. Recently, in [4] and [2] a characterization of $q-$ classical polynomials in the $q$-linear lattice $x(s)=c q^{ \pm s}+d$ is presented. We will extend these results for the $q$-quadratic lattice

$$
x(s)=c_{1} q^{s}+c_{2} q^{-s}+c_{3}, \quad q \in \widetilde{\mathbb{C}} .
$$

For our purpose, we need to redefine the concept of $q$-classical OPS. Moreover, because the measures are supported on the real line, we will consider $\Omega$ as the interval ${ }^{1}[a, b]$.

DEFINITION 4.1. The sequence $\left(p_{n}\right)$, where $p_{n}$ is a polynomial on $x(s)$ of degree $n$ for all $n \in \mathbb{N}_{0}$, is said to be a q-classical OPS on the lattice $x(s)$ if it satisfies the property of orthogonality (7) where

(i) $\rho(s)$ is a solution of the q-Pearson equation

$$
\Delta(\sigma(s) \rho(s))=\tau(s) \rho(s) \nabla x_{1}(s) .
$$

\footnotetext{
${ }^{1}$ If $|b|<|a|=\infty$ (resp. $\left.|a|<|b|=\infty\right)$ then we write $(a, b]$ (resp. $\left.[a, b)\right)$; if $|b|=|a|=\infty$ then we write $(a, b)$.
} 
(ii) $\sigma(s)+\frac{1}{2} \tau(s) \nabla x_{1}(s)$ is a polynomial on $x(s)$ of degree, at most, 2.

(iii) $\tau(s)$ is a polynomial on $x(s)$ of degree 1.

$(\sigma(s), \rho(s), x(s))$ is said to be $q$-classical if it satisfies (i)-(iii).

Notice that the above definition coincides with Definition 1.1 given in [4] for the $q$-linear lattices and generalizes it to the $q$-quadratic lattice.

LEMMA 4.1. [24, (3.2.5), p. 62] For every polynomial $\pi$ and every integer $k, \pi\left(x_{k}(s)\right)+\pi\left(x_{k}(s-\right.$ $1)$ ) is a polynomial on $x_{k-1}(s)$ of degree $\operatorname{deg}(\pi)$.

LEMMA 4.2. If $(\sigma(s), \rho(s), x(s))$ is q-classical, then for every integer $k$ and every polynomial $\pi$, the function

$$
\mathscr{R}_{1}\left(\rho_{k}(s), x_{k}(s)\right)\left(\pi\left(x_{k+1}(s)\right)\right)
$$

is a polynomial on $x_{k}(s)$ of degree $\operatorname{deg}(\pi)+1$.

Proof: By hypothesis, we know that $\widehat{\sigma}(x(s)):=\sigma(s)+\frac{1}{2} \tau(s) \nabla x_{1}(s)$ and $\tau(s)$ are polynomials on $x(s)$ of degree, at most 2 , and 1 , respectively. From a direct calculation we get that

$$
\mathscr{R}_{1}\left(\rho_{k}(s), x_{k}(s)\right)(1)=\frac{\sigma(s+k)+\tau(s+k) \nabla x_{1}(s+k)-\sigma(s)}{\nabla x_{k+1}(s)},
$$

is a polynomial on $x_{k}(s)$ of degree 1 .

Furthermore, for every integer $k$ and every polynomial $\pi$, we get

$$
\begin{aligned}
\mathscr{R}_{1}\left(\rho_{k}(s), x_{k}(s)\right)( & \left.\left(x_{k+1}(s)\right)\right)=\frac{\left(\sigma(s+k)+\tau(s+k) \nabla x_{1}(s+k)\right)}{\nabla x_{k+1}(s)} \pi\left(x_{k+1}(s)\right)-\frac{\sigma(s)}{\nabla x_{k+1}(s)} \\
& \times \pi\left(x_{k+1}(s-1)\right)=\frac{\Theta(s+k)}{\nabla x_{k+1}(s)} \pi\left(x_{k+1}(s)\right)-\frac{\sigma(s)}{\nabla x_{k+1}(s)} \pi\left(x_{k+1}(s-1)\right),
\end{aligned}
$$

being $\Theta(s):=\sigma(s)+\tau(s) \nabla x_{1}(s)$.

So, $\mathscr{R}_{1}\left(\rho_{k}(s), x_{k}(s)\right)\left(\pi\left(x_{k+1}(s)\right)\right)$ is a polynomial on $x_{k}(s)$ if and only if

$$
\frac{\Theta\left(s+\frac{k}{2}\right)}{\nabla x_{1}(s)} \pi\left(x_{1}(s)\right)-\frac{\sigma\left(s-\frac{k}{2}\right)}{\nabla x_{1}(s)} \pi\left(x_{-1}(s)\right)
$$

is a polynomial on $x(s)$. But we can rewrite this expression in the form

$$
\frac{\Theta\left(s+\frac{k}{2}\right)-\sigma\left(s-\frac{k}{2}\right)}{\nabla x_{1}(s)} \pi\left(x_{1}(s)\right)+\frac{\sigma\left(s-\frac{k}{2}\right)}{\nabla x_{1}(s)}\left(\pi\left(x_{1}(s)\right)-\pi\left(x_{-1}(s)\right)\right),
$$

or, equivalently,

$$
\frac{\Theta\left(s+\frac{k}{2}\right)-\sigma\left(s-\frac{k}{2}\right)}{\nabla x_{1}(s)} \pi\left(x_{-1}(s)\right)+\frac{\Theta\left(s+\frac{k}{2}\right)}{\nabla x_{1}(s)}\left(\pi\left(x_{1}(s)\right)-\pi\left(x_{-1}(s)\right)\right) .
$$

Taking the arithmetic mean of the above expressions, using Lemma 4.1 as well as the relation

$$
\Theta\left(s+\frac{k}{2}\right)-\sigma\left(s-\frac{k}{2}\right)=q_{1}(x(s)) \nabla x_{1}(s),
$$

where $q_{1}$ is a polynomial of degree 1 , we deduce that (16) is a polynomial on $x(s)$ of degree, at most, $\operatorname{deg}(\pi)+1$. Moreover, from a straightforward calculation the coefficient of $x^{m+1}$ is

$$
\alpha_{q}(m+2 k) \tau^{\prime}+[m+2 k]_{q} \frac{\widehat{\sigma}^{\prime \prime}}{2} \neq 0, \quad m=\operatorname{deg}(\pi),
$$

where $\widehat{\sigma}(x)=\frac{\widehat{\sigma}^{\prime \prime}}{2} x^{2}+\widehat{\sigma}^{\prime}(0) x+\widehat{\sigma}(0)$ and $\tau(x)=\tau^{\prime} x+\tau(0)$. Hence the result follows.

Taking into account all these results, we can state the preliminary results related to our main Theorem. 
THEOREM 4.1. Let $\left(p_{n}\right)$ be a q-classical OPS with respect to $\rho(s)$ on $x(s)$ such that

$$
x^{k}(a) x_{-1}^{l}(a) \sigma(a) \rho(a)=x^{k}(b) x_{-1}^{l}(b) \sigma(b) \rho(b)=0, \quad k, l \geq 0 .
$$

Then, $\left(\Delta^{(1)} p_{n+1}\right)$ is a q-classical OPS with respect to the function $\rho_{1}(s)$ on $x_{1}(s)$.

Furthermore, if the condition (17) holds then the converse is also true.

REMARK 4.1. Observe that if, for instance, $|a|=\infty$ (the case $|b|=\infty$ is analogous) the relations regarding to a into (17) need to be replaced by

$$
\lim _{t \rightarrow-\infty} x^{k}(t) x_{-1}^{l}(t) \sigma(t) \rho(t)=0, \quad k, l \geq 0 .
$$

Proof: Let $\left(p_{n}\right)$ be a $q$-classical OPS with respect to $\rho(s)$ on $x(s)$. From Lemma 4.2 the function $Q_{k}(x(s)):=\mathscr{R}_{1}(\rho(s), x(s))\left(p_{k-1}\left(x_{1}(s)\right)\right)$ is a polynomial on $x(s)$ of degree $k$. Therefore for any $n>k \geq 1$

$$
\begin{aligned}
0 & =\sum_{s=a}^{b-1} p_{n}(s) Q_{k}(s) \rho(s) \nabla x_{1}(s) \\
& =\sum_{s=a}^{b-1} p_{n}(s)\left(\mathscr{R}_{1}(\rho(s), x(s))\left(p_{k-1}\left(x_{1}(s)\right)\right)\right) \rho(s) \nabla x_{1}(s) \\
& =\sum_{s=a}^{b-1} p_{n}(s) \nabla\left(\rho_{1}(s) p_{k-1}\left(x_{1}(s)\right)\right)
\end{aligned}
$$

Taking into account the Leibniz rule, i.e.

$$
\nabla(f(s) g(s))=(\nabla f(s)) g(s)+f(s-1)(\nabla g(s)),
$$

the following formula holds

$$
\sum_{s=a}^{b-1} g(s)(\nabla f(s))=\left.f(s) g(s+1)\right|_{s=a-1} ^{s=b-1}-\sum_{s=a}^{b-1} f(s)(\Delta g(s))
$$

Thus we get

$$
\begin{aligned}
0 & =\left.p_{n}(s+1) \rho_{1}(s) p_{k-1}\left(x_{1}(s)\right)\right|_{s=a-1} ^{s=b-1}-\sum_{s=a}^{b-1}\left(\Delta p_{n}(s)\right) \rho_{1}(s) p_{k-1}\left(x_{1}(s)\right) \\
& =\left.p_{n}(s) \rho(s) \sigma(s) p_{k-1}\left(x_{-1}(s)\right)\right|_{s=a} ^{s=b}-\sum_{s=a}^{b-1}\left(\Delta p_{n}(s)\right) \rho_{1}(s) p_{k-1}\left(x_{1}(s)\right) \quad\left(\Delta x(s)=\nabla x_{2}(s)\right) \\
& =\left.p_{n}(s) \sigma(s) \rho(s) p_{k-1}\left(x_{-1}(s)\right)\right|_{s=a} ^{s=b}-\sum_{s=a}^{b-1}\left(\Delta^{(1)} p_{n}(s)\right) p_{k-1}\left(x_{1}(s)\right) \rho_{1}(s) \nabla x_{2}(s) .
\end{aligned}
$$

Hence $\left(\Delta^{(1)} p_{n+1}\right)$ is an OPS with respect to $\rho_{1}(s)$ on the lattice $x_{1}(s)$.

Now, we prove that $\rho_{1}(s)$ satisfies a $q$-Pearson equation as (15) on the lattice $x_{1}(s)$. Indeed since $\left(p_{n}\right)$ is a classical OPS we get

$$
\begin{aligned}
\frac{\Delta\left(\sigma(s) \rho_{1}(s)\right)}{\rho_{1}(s)} & =\frac{\sigma(s+1) \rho_{1}(s+1)}{\rho_{1}(s)}-\sigma(s) \\
& =\sigma(s+1)+\tau(x(s+1)) \nabla x_{1}(s+1)-\sigma(s) \\
& =\widehat{\sigma}(s+1)-\widehat{\sigma}(s)+\frac{1}{2} \tau(s+1) \nabla x_{1}(s+1)+\frac{1}{2} \tau(s) \nabla x_{1}(s),
\end{aligned}
$$

where $\widehat{\sigma}(s)=\sigma(s)+\frac{1}{2} \tau(x(s)) \nabla x_{1}(s)$ is a polynomial on $x(s)$ of degree at most 2 , and after a straightforward calculation we deduce that this expression is equal to $\widehat{\tau}_{1}\left(x_{1}(s)\right) \nabla x_{2}(s)$ with 
$\operatorname{deg}\left(\widehat{\tau_{1}}\right) \leq 1$

Moreover, using the last expressions we get

$$
\sigma(s)+\frac{1}{2} \widehat{\tau}_{1}\left(x_{1}(s)\right) \nabla x_{2}(s)=\frac{1}{2}\left(\widehat{\sigma}(s+1)+\widehat{\sigma}(s)+\frac{1}{2} \tau(s+1) \nabla x_{1}(s+1)-\frac{1}{2} \tau(s) \nabla x_{1}(s)\right),
$$

is a polynomial on $x_{1}(s)$ of degree, at most, 2 and hence the result holds.

For the converse, we know that there exist two polynomials on $x_{1}(s), \hat{\sigma}(s)$ and $\tau_{1}$, of degree at most 2 and 1, respectively, such that

$$
\Delta\left[\sigma(s) \rho_{1}(s)\right]=\tau_{1}\left(x_{1}(s)\right) \rho_{1}(s) \nabla x_{2}(s),
$$

here $\sigma(s):=\hat{\sigma}(s)-\frac{1}{2} \tau_{1}\left(x_{1}(s)\right) \nabla x_{2}(s)$.

So, we only need to check that

$$
\tau(s):=\frac{\nabla x(s)}{\nabla x_{1}(s)}\left(\tau_{1}\left(x_{1}(s-1)\right)-\frac{\nabla \sigma(s)}{\nabla x(s)}\right),
$$

and $\sigma(s)+\frac{1}{2} \tau(s) \nabla x_{1}(s)$ are polynomials on $x(s)$ of degree at most 1 and 2 , respectively, which is a direct calculation and hence the result follows.

REMARK 4.2. Notice that in the case $a=-\infty$ (the case $b=\infty$ is analogous) one should write the formula (18) as

$$
\sum_{s=-\infty}^{b-1} g(s) \nabla(f(s))=f(b-1) g(b)-\left(\lim _{t \rightarrow-\infty} f(t) g(t+1)\right)-\sum_{s=-\infty}^{b-1} f(s) \Delta(g(s)) .
$$

THEOREM 4.2. Let $\left(p_{n}\right)$ be a q-classical OPS with respect to $\rho(s)$ on $x(s)$ such that

$$
x^{k}(a) x_{-1}^{l}(a) \rho(a)=x^{k}(b) x_{-1}^{l}(b) \rho(b)=0, \quad \forall k, l=0,1, \ldots
$$

If $p_{-1}=0$, then the sequence $\left(\mathscr{R}_{n}\left(\rho_{-1}(s), x_{-1}(s)\right)(1)\right)$ is a q-classical OPS with respect to the weight function $\rho_{-1}(s)=\rho(s-1) / \sigma(s)$ on $x_{-1}(s)$. Furthermore, if the condition (17) holds then the converse is also true.

The proof follows the same steps as in Theorem 4.1 taking into account the properties of $\Delta^{(m)}$ and the basic relations of $\rho_{m}(s)$ and $x_{m}(s)$ for every integer $m$.

\section{REMARK 4.3.}

(i) The relation between the statements of Theorems 4.1 and 4.2 follows from

$$
\mathscr{R}_{1}\left(\rho_{-1}(s), x_{-1}(s)\right) \circ \mathscr{R}_{n}(\rho(s), x(s))=\mathscr{R}_{n+1}\left(\rho_{-1}(s), x_{-1}(s)\right), \quad n=0,1,2, \ldots
$$

(ii) If the property of orthogonality

$$
\sum_{s=a}^{b-1} p_{n}(x(s)) p_{m}(x(s)) \rho(s) \nabla x_{1}(s)=0,
$$

and the boundary conditions (17) holds, then for every integer $k$ we get

$$
\sum_{s=a-k}^{b-k-1}\left(\Delta^{(k)} p_{n}\left(x_{k}(s)\right)\right)\left(\Delta^{(k)} p_{m}\left(x_{k}(s)\right)\right) \rho_{k}(s) \nabla x_{k+1}(s)=0,
$$

where if $k \geq 0$

$$
\Delta^{(-k)}:=\mathscr{R}_{k}\left(\rho_{-k}(s), x_{-k}(s)\right) .
$$


Now we can state the first main result of this paper.

THEOREM 4.3. Let $\left(p_{n}\right)$ be an OPS with respect to $\rho(s)$ on the lattice $x(s)$ defined in (14) and let $\sigma(s)$ be such that (19) holds. Then the following statements are equivalent.

1. $\left(p_{n}\right)$ is q-classical.

2. The sequence $\left(\Delta^{(1)} p_{n}\right)$ is an OPS with respect to the weight function $\rho_{1}(s)=\sigma(s+1) \rho(s+1)$ where $\rho$ satisfies (15).

3. For every integer $k$, the sequence $\left(\mathscr{R}_{n}\left(\rho_{k}(s), x_{k}(s)\right)(1)\right)$ is an OPS with respect to the weight function $\rho_{k}(s)$ where $\rho_{0}(s)=\rho(s), \rho_{k}(s)=\rho_{k-1}(s+1) \sigma(s+1)$, and $\rho$ satisfies (15).

4. (Second order linear difference equation): $\left(p_{n}\right)$ satisfies the following second order linear difference equation of hypergeometric type

$$
\sigma(s) \frac{\Delta}{\nabla x_{1}(s)} \frac{\nabla p_{n}(s)}{\nabla x(s)}+\tau(s) \frac{\Delta p_{n}(s)}{\Delta x(s)}+\lambda_{n} p_{n}(s)=0,
$$

where $\widehat{\sigma}(s)=\sigma(s)+\frac{1}{2} \tau(s) \nabla x_{1}(s)$ and $\tau(s)$ are polynomials on $x(s)$ of degree at most 2 and 1 , respectively, and $\lambda_{n}$ is a constant.

5. $\left(p_{n}\right)$ can be expressed in terms of the Rodrigues Operator as follows

$$
p_{n}(s)=B_{n} \mathscr{R}_{n}(\rho(s), x(s))(1)=\frac{B_{n}}{\rho(s)} \frac{\nabla}{\nabla x_{1}(s)} \frac{\nabla}{\nabla x_{2}(s)} \cdots \frac{\nabla}{\nabla x_{n}(s)}\left(\rho_{n}(s)\right),
$$

where $B_{n}$ is a non zero constant.

6. (Second structure relation): There exist three sequences of complex numbers, $\left(e_{n}\right),\left(f_{n}\right)$, and $\left(g_{n}\right)$, such that the following relation holds for every $n \geq 0$, with the convention $p_{-1}=0$,

$$
\mathscr{M} p_{n}(x(s))=e_{n} \frac{\Delta p_{n+1}(s)}{\Delta x(s)}+f_{n} \frac{\Delta p_{n}(s)}{\Delta x(s)}+g_{n} \frac{\Delta p_{n-1}(s)}{\Delta x(s)},
$$

where $\mathscr{M}$ is the forward arithmetic mean operator:

$$
\mathscr{M} f(s):=\frac{f(s+1)+f(s)}{2},
$$

$e_{n} \neq 0, g_{n} \neq \gamma_{n}$ for all $n \geq 0$, and $\gamma_{n}$ is the corresponding coefficient of the three-term recurrence relation [24]

$$
x(s) p_{n}(s)=\alpha_{n} p_{n+1}(s)+\beta_{n} p_{n}(s)+\gamma_{n} p_{n-1}(s), \quad n \geq 0 .
$$

REMARK 4.4. If we consider a q-linear lattice, i.e. either $c_{1}=0$ or $c_{2}=0$, this result is "equivalent" to Theorem 1.3 stated in [4], because $\tau(s) \nabla x_{1}(s)$ is a polynomial on $x(s)$ of degree 2 and $x_{k}(s)=q^{\alpha_{k}} x(s)+\delta_{k}$, where $\alpha_{k}, \delta_{k}$ are independent of $s$ for every integer $k$.

Assuming the theorem proved let us state and proof the second main result.

THEOREM 4.4. Under the hypothesis of Theorem 4.3 the following statements are equivalent.

(i) $\left(p_{n}\right)$ is q-classical.

(ii) $\left(\Delta^{(1)} p_{n+1}\right)$ is a OPS. 
Proof: Of course (i) $\Rightarrow$ (ii) is a consequence of Theorem 4.1.

(ii) $\Rightarrow(\mathrm{i})$ : This proof is analogous to the proof given by W. Hahn in [15]. We know that the SPO $\left(p_{n}\right)$ satisfies the TTRR

$$
p_{n}(x(s))=\left(x(s)-a_{n}\right) p_{n-1}(x(s))-b_{n} p_{n-2}(x(s)), \quad n \geq 1,
$$

with initial conditions $p_{-1}=0$ and $p_{0}=1$. Thus applying the (forward) arithmetic mean process we get

$$
\Delta^{(1)} p_{n}(x(s))=\mathscr{M} p_{n-1}(x(s))-b_{n} \Delta^{(1)} p_{n-2}(x(s))+\left(\alpha_{q}(2) x_{1}(s)-\tilde{a}_{n}\right) \Delta^{(1)} p_{n-1}(x(s)),
$$

with $\tilde{a}_{n}=a_{n}-c_{3}(q)\left(1-\alpha_{q}(2)\right)$.

Moreover, by hypothesis, the sequence of monic polynomials $\left([n]_{q}^{-1} \Delta^{(1)} p_{n}\right)$ also satisfies a TTRR, i.e.

$$
\frac{1}{[n]_{q}} \Delta^{(1)} p_{n}(x(s))=\left(x_{1}(s)-a_{n}^{\prime}\right) \frac{1}{[n-1]_{q}} \Delta^{(1)} p_{n-1}(x(s))-\frac{b_{n}^{\prime}}{[n-2]_{q}} \Delta^{(1)} p_{n-2}(x(s)), \quad n \geq 1 .
$$

Combining (22) and (23) to eliminate $\Delta^{(1)} p_{n-2}$ (resp. $\left.\Delta^{(1)} p_{n}\right)$ we get

$$
\begin{aligned}
\left(\frac{b_{n}^{\prime}}{[n-2]_{q}}-\frac{b_{n}}{[n]_{q}}\right) \Delta^{(1)} p_{n}(x(s))= & \left(\left(\frac{\alpha_{q}(2) b_{n}^{\prime}}{[n-2]_{q}}-\frac{b_{n}}{[n-1]_{q}}\right) x_{1}(s)-\frac{b_{n}^{\prime} \tilde{a}_{n}}{[n-2]_{q}}+\frac{b_{n} a_{n}^{\prime}}{[n-1]_{q}}\right) \Delta^{(1)} p_{n-1}(x(s)) \\
& +\frac{b_{n}^{\prime}}{[n-2]_{q}} \mathscr{M} p_{n-1}(x(s)),
\end{aligned}
$$

and

$$
\begin{aligned}
\frac{1}{[n]_{q}} \mathscr{M} p_{n-1}(x(s))= & \left(\frac{[n]_{q}-[n-2]_{q}}{2[n-1]_{q}[n]_{q}} x_{1}(s)-\frac{a_{n}^{\prime}}{[n-1]_{q}}+\frac{\tilde{a}_{n}}{[n]_{q}}\right) \Delta^{(1)} p_{n-1}(x(s)) \\
& -\left(\frac{b_{n}^{\prime}}{[n-2]_{q}}-\frac{b_{n}}{[n]_{q}}\right) \Delta^{(1)} p_{n-2}(x(s)) .
\end{aligned}
$$

Setting $n+1$ instead of $n$ in the above expression the following expression fulfills:

$$
\begin{aligned}
\frac{1}{[n+1]_{q}} \mathscr{M} p_{n}(x(s))= & \left(\frac{[n+1]_{q}-[n-1]_{q}}{2[n]_{q}[n+1]_{q}} x_{1}(s)-\frac{a_{n+1}^{\prime}}{[n]_{q}}+\frac{\tilde{a}_{n+1}}{[n+1]_{q}}\right) \Delta^{(1)} p_{n}(x(s)) \\
& +\left(\frac{b_{n+1}}{[n+1]_{q}}-\frac{b_{n+1}^{\prime}}{[n-1]_{q}}\right) \Delta^{(1)} p_{n-1}(x(s)) .
\end{aligned}
$$

In order to simplify further calculations in this proof we denote by $e_{n}:=\frac{b_{n}^{\prime}}{[n-2]_{q}}-\frac{b_{n}}{[n]_{q}}, k(x ; n)=$ $\frac{[n+1]_{q}-[n-1]_{q}}{2\left[n_{q}\right][n+1]_{q}} x-\frac{a_{n+1}^{\prime}}{[n]}+\frac{\tilde{a}_{n+1}}{[n+1]_{q}}$ and by $l(x ; n)=\left(\frac{\alpha_{q}(2) b_{n}^{\prime}}{[n-2]_{q}}-\frac{b_{n}}{[n-1]_{q}}\right) x-\frac{b_{n}^{\prime} \tilde{a}_{n}}{[n-2]_{q}}+\frac{b_{n} a_{n}^{\prime}}{[n-1]_{q}}$.

Taking into account $(24)$ and $(25)$ in order to eliminate $\Delta^{(1)} p_{n}$ we obtain

$\frac{e_{n}}{[n+1]_{q}} \mathscr{M} p_{n}(x(s))=\frac{b_{n}^{\prime}}{[n-2]_{q}} k\left(x_{1}(s) ; n\right) \mathscr{M} p_{n-1}(x(s))+k\left(x_{1}(s) ; n\right)\left(l\left(x_{1}(s) ; n\right)-e_{n+1} e_{n}\right) \Delta^{(1)} p_{n-1}(x(s))$.

By using (25) and (26) to eliminate $p_{n}$ we get

$$
\begin{gathered}
\frac{\mathscr{M}\left(l\left(x_{-1}(s) ; n\right) \nabla p_{n-1}(x(s))\right)}{\nabla x_{1}(s)}+\frac{b_{n}^{\prime}}{[n-2]_{q}} \frac{\mathscr{M}\left((\nabla x(s)) \mathscr{M} p_{n-1}(x(s-1))\right)}{\nabla x_{1}(s)} \\
=\frac{[n+1]_{q} b_{n}^{\prime}}{[n-2]_{q}} \frac{\nabla\left(k\left(x_{1}(s) ; n\right) \mathscr{M} p_{n-1}(x(s))\right)}{\nabla x_{1}(s)}+[n+1]_{q} \frac{\nabla\left(\left(l\left(x_{1}(s) ; n\right) k\left(x_{1}(s) ; n\right)-e_{n} e_{n+1}\right) \Delta^{(1)} p_{n-1}(x(s))\right)}{\nabla x_{1}(s)} .
\end{gathered}
$$

On the other hand, combining (24) and (25) to eliminate $\Delta^{(1)} p_{n-1}$ we get

$$
\frac{b_{n}^{\prime} e_{n+1}}{[n-2]_{q}} \mathscr{M} p_{n-1}(x(s))=\frac{l\left(x_{1}(s) ; n\right)}{[n+1]_{q}} \mathscr{M} p_{n}(x(s))-\left(k\left(x_{1}(s) ; n\right) l\left(x_{1}(s) ; n\right)-e_{n} e_{n+1}\right) \Delta^{(1)} p_{n}(x(s)) .
$$


By using the expressions (25) and (28) to eliminate $p_{n-1}$ we get

$$
\begin{gathered}
\frac{1}{[n+1]_{q}} \frac{\mathscr{M}\left((\nabla x(s)) \mathscr{M} p_{n}(x(s-1))\right)}{\nabla x_{1}(s)}-\frac{\mathscr{M}\left(k\left(x_{-1}(s) ; n\right) \nabla p_{n}(x(s))\right)}{\nabla x_{1}(s)} \\
=\frac{[n-2]_{q}}{b_{n}^{\prime}[n+1]_{q}} \frac{\nabla\left(l\left(x_{1}(s) ; n\right) \mathscr{M} p_{n}(x(s))\right)}{\nabla x_{1}(s)}-\frac{[n-2]_{q}}{b_{n}^{\prime}} \frac{\nabla\left(\left(l\left(x_{1}(s) ; n\right) k\left(x_{1}(s) ; n\right)-e_{n} e_{n+1}\right) \Delta^{(1)} p_{n}(x(s))\right)}{\nabla x_{1}(s)} .
\end{gathered}
$$

The following result has a technical role and it will be used in to proof of this Theorem.

LEMMA 4.3. For any two polynomials $\pi$ and $\xi$, the following relation holds:

$$
\begin{aligned}
\frac{\mathscr{M}\left(\pi\left(x_{-1}(s)\right) \nabla \xi(x(s))\right)}{\nabla x_{1}(s)} & =\frac{\nabla\left(\pi\left(x_{1}(s)\right) \mathscr{M} \xi(x(s))\right)}{\nabla x_{1}(s)}+\left(\nabla^{(1)} \pi\left(x_{1}(s)\right)\right) \xi(x(s)) \\
& =\frac{\mathscr{M}\left(\pi\left(x_{-1}(s)\right) \nabla x(s)\right)}{\nabla x_{1}(s)} \mathscr{M} \frac{\nabla \xi(x(s))}{\nabla x(s)}+\nabla\left(\pi\left(x_{1}(s)\right) \Delta x(s)\right) \frac{\nabla \Delta^{(1)} \xi(x(s))}{\nabla x_{1}(s)} .
\end{aligned}
$$

Moreover, the functions $\frac{\mathscr{M}\left(\pi\left(x_{-1}(s)\right) \nabla x(s)\right)}{\nabla x_{1}(s)}$ and $\nabla\left(\pi\left(x_{1}(s)\right) \Delta x(s)\right)$ are polynomials on $x(s)$.

The proof of this result is straightforward and we leave it as an exercise for the reader.

Therefore, using Lemma 4.3 and doing some extra calculations, we obtain that the expressions (27) and (29) become the following two second order homogeneous linear difference equations:

$$
\begin{aligned}
& \phi_{2}(x(s), n) \nabla^{(1)} \Delta^{(1)} p_{n}(x(s))+\phi_{1}(x(s), n) \mathscr{M} \frac{\nabla p_{n}(x(s))}{\nabla x(s)}+\phi_{0}(n) p_{n}(x(s))=0, \\
& \phi_{2}(x(s), n) \nabla^{(1)} \Delta^{(1)} p_{n-1}(x(s))+\phi_{1}(x(s), n) \mathscr{M} \frac{\nabla p_{n-1}(x(s))}{\nabla x(s)}+\tilde{\phi}_{0}(n) p_{n-1}(x(s))=0,
\end{aligned}
$$

where the indices indicate the degree of the polynomial coefficients.

Moreover, replacing $n$ by $n+1$ in the last expression we obtain the second order linear difference equation:

$$
\phi_{2}(x(s), n+1) \nabla^{(1)} \Delta^{(1)} p_{n}(x(s))+\phi_{1}(x(s), n+1) \mathscr{M} \frac{\nabla p_{n}(x(s))}{\nabla x(s)}+\tilde{\phi}_{0}(n+1) p_{n}(x(s))=0 .
$$

So, we have two difference equations for $p_{n}$ which only differ in a constant factor.

Therefore $p_{n}$ satisfies the following two second order linear difference equations:

$$
\begin{aligned}
& \varphi_{2}(x(s)) \nabla^{(1)} \Delta^{(1)} p_{n}(x(s))+\varphi_{1}(x(s)) \mathscr{M} \frac{\nabla p_{n}(x(s))}{\nabla x(s)}+\varphi_{0}(x(s)) p_{n}(x(s))=0, \\
& \psi_{2}(x(s)) \nabla^{(1)} \Delta^{(1)} p_{n}(x(s))+\psi_{1}(x(s)) \mathscr{M} \frac{\nabla p_{n}(x(s))}{\nabla x(s)}+\psi_{0}(x(s)) p_{n}(x(s))=0,
\end{aligned}
$$

where, again, the indices indicate the degree of the polynomial coefficients. Denoting by $\sigma(s):=$ $\varphi_{2}(x(s))+\varphi_{1}(x(s)) \nabla x_{1}(s), \zeta(s):=\psi_{2}(x(s))+\psi_{1}(x(s)) \nabla x_{1}(s)$, the above expressions can be rewritten as

$$
\begin{aligned}
& \sigma(s) \nabla^{(1)} \Delta^{(1)} p_{n}(x(s))+\varphi_{1}(x(s)) \Delta^{(1)} p_{n}(x(s))+\varphi_{0}(n) p_{n}(x(s))=0, \\
& \zeta(s) \nabla^{(1)} \Delta^{(1)} p_{n}(x(s))+\psi_{1}(x(s)) \Delta^{(1)} p_{n}(x(s))+\psi_{0}(n) p_{n}(x(s))=0 .
\end{aligned}
$$

Notice that $\sigma$ and $\zeta$ are not always polynomials on $x(s)$.

So,

$$
\begin{aligned}
& \left(\varphi_{1}(x(s)) \zeta(s)-\sigma(s) \psi_{1}(x(s))\right) \Delta^{(1)} p_{n}(x(s)) \\
& +\left(\varphi_{0}(n) \zeta(s)-\sigma(s) \psi_{0}(n)\right) p_{n}(x(s))=0 .
\end{aligned}
$$

If $n>3$, we get that $p_{n}$ and $\Delta^{(1)} p_{n}$, has a common zero but $p_{n}$ has not no multiple zeros. Hence, after dividing by the common factor, the polynomial coefficients of the difference equations for 
$p_{n+1}, p_{n}$, and $p_{n-1}$ are the same. Taking this into account follow the coefficients do not depend on $n$, so $p_{n}(x)$ satisfies the second order linear difference equation

$$
\phi(x(s)) \nabla^{(1)} \Delta^{(1)} p_{n}(x(s))+\psi(x(s)) \mathscr{M} \frac{\nabla p_{n}(x(s))}{\nabla x(s)}+\lambda_{n} p_{n}(x(s))=0,
$$

where $\operatorname{deg} \phi \leq 2, \operatorname{deg} \psi \leq 1$, and $\lambda_{n}=-[n]_{q}\left(\alpha_{q}(n-1) b_{\psi}+[n-1] a_{\phi}\right)$ depends on $n$ since $p_{n}$ is a polynomial, being $a_{\phi}$ and $b_{\psi}$ the leading coefficients of $\phi$ and $\psi$, respectively.

Hence, according to the 4 th condition of Theorem $4.3,\left(p_{n}\right)$ is $q$-classical.

Let us prove now Theorem 4.3. From Theorem 4.1, Theorem 4.2, and its Corollary, as well as Remark 4.3 we know that $(1) \rightarrow(2) \rightarrow(3) \rightarrow(1)$.

PROPOSITION 4.1. ((1) $\rightarrow(4))$ If $\left(p_{n}\right)$ is a q-classical OPS with respect to $\rho(s)$ satisfying the boundary relations $(19)$ then $\left(p_{n}\right)$ satisfies the second order linear difference equation of hypergeometric type (20).

Proof: By Lemma 4.2 the function $\mathscr{R}_{1}(\rho(s), x(s))\left(\Delta^{(1)} p_{n}(x(s))\right.$ is a polynomial on $x(s)$ of degree $n$. On the other hand, by Theorem $4.1\left(\Delta^{(1)} p_{n}\right)$ is a $q$-classical OPS with respect to $\rho_{1}(s)$ on $x_{1}(s)$. Thus, for $0 \leq k<n$,

$$
\begin{aligned}
& \sum_{s=a}^{b-1} Q_{k}(x(s)) \mathscr{R}_{1}(\rho(s), x(s))\left(\Delta^{(1)} p_{n}(x(s))\right) \rho(s) \nabla x_{1}(s) \\
= & \sum_{s=a}^{b-1} Q_{k}(x(s)) \nabla\left(\rho_{1}(s) \Delta^{(1)} p_{n}(x(s))\right) \\
\stackrel{(18)}{=} & \left.Q_{k}(x(s)) \sigma(s) \rho(s) \Delta^{(1)} p_{n}(x(s-1))\right|_{s=a} ^{s=b}-\sum_{s=a}^{b-1} \Delta^{(1)}\left(p_{n}(x(s))\right) \Delta^{(1)}\left(Q_{k}(x(s))\right) \rho_{1}(s) \nabla x_{2}(s) .
\end{aligned}
$$

By hypothesis and Theorem 4.1 the last expression vanishes and, as a consequence, there exists a non zero constant $\lambda_{n}$, independent of $s$, such that

$$
\mathscr{R}_{1}(\rho(s), x(s))\left(\Delta^{(1)} p_{n}(x(s))\right)=-\lambda_{n} p_{n}(x(s)),
$$

since $\left(p_{n}\right)$ is an OPS with respect to $\rho(s)$ on $x(s)$.

Finally, using that the above expression is indeed $\mathscr{H}_{q}$ (see (13)) it is clear that the above expression (20) and hence the result holds.

$(4) \rightarrow(5)$ : This is a well-known property. See, for instance, $\S 3.2 .2$ in page 64 of [24].

$(5) \rightarrow(1)$ : By setting $n=1$ in the Rodrigues formula we obtain the Pearson type equation (15) that the weight function $\rho(s)$ satisfies.

PROPOSITION 4.2. $((6) \leftrightarrow(1))$. Let $\left(p_{n}\right)$ be an OPS with respect to $\rho(s)$ on the lattice $x(s)$ defined in (14) and let $\sigma(s)$ be such that (19) holds. The sequence $\left(p_{n}\right)$ is q-classical if and only if there exist sequences of complex numbers, $\left(e_{n}\right),\left(f_{n}\right)$, and $\left(g_{n}\right)$, such that the following relation holds

$$
\mathscr{M} p_{n}(x(s))=e_{n} \frac{\Delta p_{n+1}(x(s))}{\Delta x(s)}+f_{n} \frac{\Delta p_{n}(x(s))}{\Delta x(s)}+g_{n} \frac{\Delta p_{n-1}(x(s))}{\Delta x(s)},
$$

with the convention $p_{-1}=0$, where $e_{n} \neq 0, g_{n} \neq \gamma_{n}$ for all $n \geq 0$.

Proof: If $\left(p_{n}\right)$ is an OPS then it satisfies a TTRR, i.e. there exist three sequences of complex numbers, $\left(\alpha_{n}\right),\left(\beta_{n}\right)$, and $\left(\gamma_{n}\right)$, with $\gamma_{n} \neq 0$, such that

$$
x(s) p_{n}(x(s))=\alpha_{n} p_{n+1}(x(s))+\beta_{n} p_{n}(x(s))+\gamma_{n} p_{n-1}(x(s)), \quad n \geq 0 .
$$

If $\left(p_{n}\right)$ is $q$-classical, then $\left(\Delta^{(1)} p_{n+1}\right)$ is an OPS, and therefore there exist sequences of complex numbers, $\left(\alpha_{n}^{(1)}\right),\left(\beta_{n}^{(1)}\right)$, and $\left(\gamma_{n}^{(1)}\right)$, with $\gamma_{n}^{(1)} \neq 0$, such that

$$
x_{1}(s) \Delta^{(1)} p_{n}(x(s))=\alpha_{n}^{(1)} \Delta^{(1)} p_{n+1}(x(s))+\beta_{n}^{(1)} \Delta^{(1)} p_{n}(x(s))+\gamma_{n}^{(1)} \Delta^{(1)} p_{n-1}(x(s)) .
$$


But

$$
\Delta^{(1)}\left(x(s) p_{n}(x(s))\right)=(\mathscr{M} x(s)) \Delta^{(1)} p_{n}(x(s))+\mathscr{M} p_{n}(x(s)) .
$$

So combining (31), (32), and (33) we get

$$
\mathscr{M} p_{n}(x(s))=\left(\alpha_{n}-\frac{[2]_{q}}{2} \alpha_{n}^{(1)}\right) \Delta^{(1)} p_{n+1}(x(s))+\widehat{\beta}_{n} \Delta^{(1)} p_{n}(x(s))+\left(\gamma_{n}-\frac{[2]_{q}}{2} \gamma_{n}^{(1)}\right) \Delta^{(1)} p_{n-1}(x(s)),
$$

where

$$
\widehat{\beta}_{n}=\left(\beta_{n}-c_{3}\right)-\frac{[2]_{q}}{2}\left(\beta_{n}^{(1)}-c_{3}\right) .
$$

Moreover, the coefficient of $\Delta^{(1)} p_{n-1}$ is different from $\gamma_{n}$ because $\gamma_{n}^{(1)} \neq 0$.

Conversely, if there exist sequences of complex numbers, $\left(e_{n}\right),\left(f_{n}\right)$, and $\left(g_{n}\right)$, such that the following relation holds

$$
\mathscr{M} p_{n}(x(s))=e_{n} \frac{\Delta p_{n+1}(x(s))}{\Delta x(s)}+f_{n} \frac{\Delta p_{n}(x(s))}{\Delta x(s)}+g_{n} \frac{\Delta p_{n-1}(x(s))}{\Delta x(s)}, \quad n \geq 0,
$$

assuming $p_{-1}=0$, then from (33) we get

$\frac{[2]_{q}}{2} x_{1}(s) \Delta^{(1)} p_{n}(x(s))=\left(\alpha_{n}-e_{n}\right) \Delta^{(1)} p_{n+1}(x(s))+\widehat{\beta}_{n}^{(1)} \Delta^{(1)} p_{n}(x(s))+\left(\gamma_{n}-g_{n}\right) \Delta^{(1)} p_{n-1}(x(s))$,

where

$$
\widehat{\beta}_{n}^{(1)}=\beta_{n}-c_{3}-f_{n}+\frac{[2]_{q}}{2} c_{3}
$$

By hypothesis $g_{n} \neq \gamma_{n}$, hence by Favard's Theorem $\left(\Delta^{(1)} p_{n+1}\right)$ is an OPS, and by Hahn's Theorem the result holds.

\section{The examples}

\subsection{The Askey-Wilson Polynomials}

The Askey-Wilson polynomials, which were introduced by R. Askey and J. Wilson, are located in the top of the $q$-Askey tableau [16]. These polynomials can be written as a basic hypergeometric series

$$
p_{n}(x(s) ; a, b, c, d \mid q)=\frac{(a b ; q)_{n}(a c ; q)_{n}(a d ; q)_{n}}{a^{n}}{ }_{4} \varphi_{3}\left(\begin{array}{c}
q^{-n}, a b c d q^{n-1}, a q^{s}, a q^{-s} \\
a b, a c, a d
\end{array} \mid q ; q\right),
$$

where $a, b, c, d, a b, a c, a d, b c, b d, c d \notin\left\{q^{m}: m \in \mathbb{Z}\right\}$.

REMARK 5.1. Notice that although this family does not satisfy a property of orthogonality (7) it is orthogonal with respect the linear functional $\mathbf{u}^{A W}$ which has the following integral representation [16, (3.1.2), p.63]

$$
\left\langle\mathbf{u}^{A W}, p\right\rangle=\int_{-1}^{1} p(x) \widetilde{\omega}(x) d x=\int_{-1}^{1} \frac{p(x)}{\sqrt{1-x^{2}}}\left|\frac{\left(e^{2 i \theta} ; q\right)_{\infty}}{\left(a e^{i \theta}, b e^{i \theta}, c e^{i \theta}, d e^{i \theta} ; q\right)_{\infty}}\right|^{2} d x, \quad x=\cos \theta .
$$

Furthermore, such a functional fulfills the distributional equation

$$
\Delta^{(1)}\left(\sigma^{A W} \mathbf{u}^{A W}\right)=p_{1}(x(s) ; a, b, c, d \mid q) \mathbf{u}^{A W},
$$

and it is straightforward to check that $\left(\sigma^{A W}(s), \omega(s), x(s)\right)$ is q-classical, where $x(s)=\frac{1}{2}\left(q^{s}+q^{-s}\right)$, i.e. $c_{1}=c_{2}=\frac{1}{2}$ and $c_{3}=0, \sigma^{A W}(s)=-\kappa_{q}^{2} q^{-2 s+\frac{1}{2}}\left(q^{s}-a\right)\left(q^{s}-b\right)\left(q^{s}-c\right)\left(q^{s}-d\right)$, and hence we can apply Theorem 4.3 to Askey-Wilson polynomials. 
These polynomials are the polynomial eigenfunctions of the linear difference operator [16, (3.1.6)]

$$
\mathscr{H}_{q}^{A W}=\frac{1}{\nabla x_{1}(s)}\left(\sigma^{A W}(-s) \frac{\Delta}{\Delta x(s)}-\sigma^{A W}(s) \frac{\nabla}{\nabla x(s)}\right) .
$$

Notice that if $\tau^{A W}(x(s))=p_{1}(x(s) ; a, b, c, d \mid q)$ a straightforward calculation yields to

$$
\sigma^{A W}(-s)=\sigma^{A W}(s)+\tau^{A W}(x(s)) \nabla x_{1}(s) .
$$

Here, as before, $\kappa_{q}=q^{\frac{1}{2}}-q^{-\frac{1}{2}}$. With these parameters, let us consider the following function

$$
\rho^{A W}(s)=q^{-2 s^{2}}(a, b, c, d ; q)_{s}(a, b, c, d ; q)_{-s},
$$

where $(a ; q)_{0}=1,(a ; q)_{k}=(1-a)(1-a q) \cdots\left(1-a q^{k-1}\right), k \geq 1$, and

$$
(a ; q)_{-k}=\frac{1}{\left(1-a q^{-1}\right)\left(1-a q^{-2}\right) \cdots\left(1-a q^{-k}\right)}, \quad k \geq 1,
$$

LEMMA 5.1. The function $\rho^{A W}(s)$ satisfies the Pearson-type equation

$$
\sigma^{A W}(s+1) \rho(s+1)=\sigma^{A W}(-s) \rho(s) .
$$

\section{Proof:}

$$
\begin{aligned}
\frac{\rho^{A W}(s+1)}{\rho^{A W}(s)} & =\frac{q^{-2(s+1)^{2}}}{q^{-2 s^{2}}} \frac{(a, b, c, d ; q)_{s+1}(a, b, c, d ; q)_{-s-1}}{(a, b, c, d ; q)_{s}(a, b, c, d ; q)_{-s}} \\
& =\frac{q^{-4 s-2}\left(1-a q^{s}\right)\left(1-b q^{s}\right)\left(1-c q^{s}\right)\left(1-d q^{s}\right)}{\left(1-a q^{-s-1}\right)\left(1-b q^{-s-1}\right)\left(1-c q^{-s-1}\right)\left(1-d q^{-s-1}\right)} \\
& =\frac{q^{2 s}\left(q^{-s}-a\right)\left(q^{-s}-b\right)\left(q^{-s}-c\right)\left(q^{-s}-d\right)}{q^{-2 s-2}\left(q^{s+1}-a\right)\left(q^{s+1}-b\right)\left(q^{s+1}-c\right)\left(q^{s+1}-d\right)}=\frac{\sigma^{A W}(-s)}{\sigma^{A W}(s+1) .}
\end{aligned}
$$

Notice that from (34), the equation (35) becomes the Pearson-type equation (15).

Taking into account that $\rho_{n}(s)=\rho_{n-1}(s+1) \sigma(s+1), n \geq 1$, with $\rho_{0}(s)=\rho(s)$, a straightforward calculation gives

$$
\rho_{n}^{A W}(s)=\kappa_{q}^{2 n} q^{-2 s^{2}-2 s n-n^{2}+\frac{3}{2} n}(a, b, c, d ; q)_{s+n}(a, b, c, d ; q)_{-s} .
$$

Then the Askey-Wilson polynomials can be written for every nonnegative integer $n$ as

$$
p_{n}(x(s) ; a, b, c, d \mid q)=\frac{B_{n} \kappa_{q}^{2 n} q^{2 s^{2}}}{(a, b, c, d ; q)_{s}(a, b, c, d ; q)_{-s}} \nabla^{(n)} \frac{(a, b, c, d ; q)_{s+n}(a, b, c, d ; q)_{-s}}{q^{2 s^{2}+2 s n+n^{2}-\frac{3}{2} n}},
$$

where $B_{n}=2^{-n} \kappa_{q}^{-n} q^{\frac{n(3 n-5)}{4}}$.

Furthermore, since this family fulfills the following difference relation [16, (3.1.8)]

$$
\Delta^{(1)} p_{n}(x(s) ; a, b, c, d \mid q)=2[n]_{q}\left(1-a b c d q^{n-1}\right) p_{n-1}\left(x_{1}(s) ; a q^{\frac{1}{2}}, b q^{\frac{1}{2}}, c q^{\frac{1}{2}}, d q^{\frac{1}{2}} \mid q\right),
$$

and the coefficients of the second structure relation are

$$
\begin{aligned}
e_{n}= & \frac{2[n]_{q}\left(1-a b c d q^{n-1}\right)^{2}-[2]_{q}[n+1]_{q}\left(1-a b c d q^{n}\right)^{2}}{4[n]_{q}\left(1-a b c d q^{n-1}\right)\left(1-a b c d q^{2 n-1}\right)\left(1-a b c d q^{2 n}\right)} \\
f_{n}= & \frac{1-q}{4}\left(a-a^{-1} q^{-1}\right)-\frac{1}{2}\left(A_{n}(a, b, c, d \mid q)+C_{n}(a, b, c, d \mid q)\right. \\
& \left.-\frac{[2]_{q}}{2}\left(A_{n-1}\left(a q^{\frac{1}{2}}, b q^{\frac{1}{2}}, c q^{\frac{1}{2}}, d q^{\frac{1}{2}} \mid q\right)+C_{n-1}\left(a q^{\frac{1}{2}}, b q^{\frac{1}{2}}, c q^{\frac{1}{2}}, d q^{\frac{1}{2}} \mid q\right)\right)\right), \\
g_{n}= & \frac{\left(1-a b c d q^{[n-2]_{q}}\right)\left(1-a b c d q^{2 n-2}\right)\left(1-a b c d q^{2 n-1}\right)}{4[n]_{q}\left(1-a b c d q^{n-1}\right)^{2}}\left(\frac{2[n]_{q}\left(A_{n-1} C_{n}\right)(a, b, c, d \mid q)}{\left(1-a b c d q^{[n-2]_{q}}\right)^{2}}\right. \\
& \left.-\frac{[2]_{q}[n-1]_{q}\left(A_{[n-2]_{q}} C_{n-1}\right)\left(a q^{\frac{1}{2}}, b q^{\frac{1}{2}}, c q^{\frac{1}{2}}, d q^{\frac{1}{2}} \mid q\right)}{\left(1-a b c d q^{n-1}\right)^{2}}\right) .
\end{aligned}
$$


REMARK 5.2. Notice that in [19] T.H. Koornwinder has obtained a different explicit structure relation for Askey-Wilson polynomials by using the difference operator theory.

\subsection{The $q$-RACAH POLYNOMials}

We consider the $q$-Racah polynomials $u_{n}^{\alpha, \beta}(x(s), a, b)_{q}$ on the lattice

$$
x(s)=[s]_{q}[s+1]_{q}=q^{\frac{1}{2}} \kappa_{q}^{-2} q^{s}+q^{-\frac{1}{2}} \kappa_{q}^{-2} q^{-s}-[2]_{q} \kappa_{q}^{-2} .
$$

They were introduced in $[20,24]$ and deeply studied in [6]. These polynomials can be written in terms of a basic hypergeometric series [6] as follows

$$
u_{n}^{\alpha, \beta}(x(s), a, b)_{q}=D_{n 4} \varphi_{3}\left(\begin{array}{c}
q^{-n}, q^{\alpha+\beta+n+1}, q^{a-s}, q^{a+s+1} \\
q^{a-b+1}, q^{\beta+1}, q^{a+b+\alpha+1}
\end{array} \mid q, q\right),
$$

where

$$
D_{n}=\frac{q^{-\frac{n}{2}(2 a+\alpha+\beta+n+1)}\left(q^{a-b+1} ; q\right)_{n}\left(q^{\beta+1} ; q\right)_{n}\left(q^{a+b+\alpha+1} ; q\right)_{n}}{\kappa_{q}^{2 n}(q ; q)_{n}} .
$$

Observe that from the above formulas the polynomials $u_{n}^{\alpha, \beta}(x(s), a, b)_{q}$ are multiples of the standard $q$-Racah polynomials $\mathscr{R}_{n}\left(\mu\left(q^{b+s}\right) ; q^{\alpha}, q^{\beta}, q^{a-b}, q^{-a-b} \mid q\right)$.

These polynomials are the polynomial eigenfunctions of the second order homogeneous linear difference hypergeometric operator

$$
\mathscr{H}_{q}^{q R}=\frac{1}{\nabla x_{1}(s)}\left(\sigma^{q R}(-s-1) \frac{\Delta}{\Delta x(s)}-\sigma^{q R}(s) \frac{\nabla}{\nabla x(s)}\right),
$$

where

$$
\sigma^{q R}(s)=-\frac{q^{-2 s}}{\kappa_{q}^{4} q^{\frac{\alpha+\beta}{2}}}\left(q^{s}-q^{a}\right)\left(q^{s}-q^{-b}\right)\left(q^{s}-q^{\beta-a}\right)\left(q^{s}-q^{b+\alpha}\right)=[s-a]_{q}[s+b]_{q}[s+a-\beta]_{q}[b+\alpha-s]_{q} .
$$

Furthermore, taking into account the $q$-difference relation

$$
\Delta^{(1)} u_{n}^{\alpha, \beta}(x(s), a, b)_{q}=[\alpha+\beta+n+1]_{q} u_{n-1}^{\alpha+1, \beta+1}\left(x_{1}(s), a+\frac{1}{2}, b-\frac{1}{2}\right)_{q} .
$$

The coefficients for the second structure relation are

$$
\begin{aligned}
e_{n}= & \frac{2[n+\alpha+\beta+1]_{q}-[2]_{q}[n+\alpha+\beta+2]_{q}}{2[2 n+\alpha+\beta+1]_{q}[2 n+\alpha+\beta+2]_{q}}[n+1]_{q}, \\
f_{n}= & \beta_{n}(a, b, \alpha, \beta)-\frac{[2]_{q}}{2} \beta_{n}\left(a+\frac{1}{2}, b-\frac{1}{2}, \alpha+1, \beta+1\right), \\
g_{n}= & \frac{[a+b+\alpha+n]_{q}[a+b-\beta-n]_{q}[\alpha+n]_{q}[\beta+n]_{q}[b-a+\alpha+\beta+n]_{q}[b-a-n]_{q}}{2[\alpha+\beta+2 n]_{q}[\alpha+\beta+2 n+1]_{q}[n+\alpha+\beta+1]_{q}} \\
& \times\left(2[n+\alpha+\beta+1]_{q}-[2]_{q}[n+\alpha+\beta]_{q}\right) .
\end{aligned}
$$

\subsection{The $q$-MeIXner POLYNOMIALS}

This family of $q$-polynomials has the following representation as a basic hypergeometric series

$$
M_{n}(x ; b, c ; q)=\frac{(b q ; q)_{n}(-c)^{n}}{q^{n^{2}}}{ }_{2} \varphi_{1}\left(\begin{array}{c}
q^{-n}, x \\
b q
\end{array} \mid q ;-\frac{q^{n+1}}{c}\right), \quad x(s)=q^{-s} \equiv x
$$


They are the polynomial eigenfunctions of the linear difference operator of hypergeometric type $[16,(3.13 .5)]$

$$
\mathscr{H}_{q}^{q M}=\frac{1}{\nabla x_{1}(s)}\left((x-1)(x+b c) \frac{\Delta}{\Delta x(s)}-q^{-1} c(x-b q) \frac{\nabla}{\nabla x(s)}\right)
$$

In this case

$$
\begin{aligned}
& e_{n}=1-\frac{[2]_{q}[n+1]_{q}}{2[n]_{q}} q^{-\frac{1}{2}} \\
& f_{n}=\frac{1-q}{2}+\frac{q^{-2 n-1}}{2}\left(c-c b q^{n+1}\right)\left(2-q-q^{2}\right)+\frac{q^{-2 n}}{2}\left(c+q^{n}\right)\left(2-q-q^{2}+q^{n}-q^{n-1}\right), \\
& g_{n}=\frac{c q^{-4 n+1}\left(1-b q^{n}\right)\left(c+q^{n}\right)}{2[n]_{q}}\left(2[n]_{q}\left(1-q^{n}\right)-\left(q+q^{2}\right)[n-1]_{q}\left(1-q^{n-1}\right)\right) .
\end{aligned}
$$

\subsection{The Al-Salam \& Carlitz polynomials I AND II}

The Al-Salam \& Carlitz polynomials I (and II) appear in certain models of $q$-harmonic oscillator, see e.g. $[8,9,10,23]$. They are polynomials orthogonal on the $q$-linear lattice $x(s)=q^{s} \equiv x$, defined [16] by

$$
U_{n}^{(a)}(x ; q)=(-a)^{n} q^{\left(\begin{array}{c}
n \\
2
\end{array}\right)}{ }_{2} \varphi_{1}\left(\begin{array}{c}
q^{-n}, x^{-1} \\
0
\end{array} \mid q ; \frac{q x}{a}\right) .
$$

These polynomials are the polynomial eigenfunctions of the linear difference operator of hypergeometric type $[16,(3.24 .5)]$

$$
\mathscr{H}_{q}^{A C I}=\frac{1}{\nabla x_{1}(s)}\left(a \frac{\Delta}{\Delta x(s)}-(x-1)(x-a) \frac{\nabla}{\nabla x(s)}\right),
$$

In this case $[16,(3.24 .7)] \Delta^{(1)} U_{n}^{(a)}(x ; q)=q^{\frac{n-1}{2}}[n]_{q} U_{n-1}^{(a)}(x ; q)$, so the second structure relation coefficients are

$$
\begin{gathered}
e_{n}=1-\frac{(1+q)[n+1]_{q}}{2 q[n]_{q}}, f_{n}=(1+a) q^{n}\left(1-\frac{[2]_{q}}{2} q^{-\frac{3}{2}}\right) \\
g_{n}=\frac{a q^{n-\frac{5}{2}}}{2[n]_{q}}\left(2[n]_{q^{\frac{3}{2}}}\left(q^{n}-1\right)-[2]_{q}[n-1]_{q}\left(q^{n-1}-1\right)\right) .
\end{gathered}
$$

Taking into account that $[16$, p. 115]

$$
V_{n}^{(a)}(x ; q)=U_{n}^{(a)}\left(x ; q^{-1}\right),
$$

all the information related to the Al-Salam \& Carlitz polynomials II can be deduced from the information for the Al-Salam \& Carlitz polynomials I in a simple way by changing $q$ to $q^{-1}$.

\section{Acknowledgements}

The work of the authors has been supported by Dirección General de Investigación, Ministerio de Educación y Ciencia of Spain under grant MTM 2006-13000-C03-02. Finally, we thank the anonymous referees for the careful revision of the manuscript. Their comments and suggestions have substantially improved the presentation of the manuscript.

\section{References}

1. W. A. Al-Salam and T. S. Chihara. Another characterization of the classical orthogonal polynomials. SIAM J. Math. Anal. 3 , 65-70 (1992) 
2. M. Alfaro and R. Álvarez-Nodarse. A characterization of the classical orthogonal discrete and $q$-polynomials. J. Comput. Appl. Math. 201, 48-54 (2007)

3. R. Álvarez-Nodarse. Polinomios hipergeométricos y q-polinomios, volume 26 of Monografías del Seminario Matemático "Garcia de Galdeano". Prensas Universitarias de Zaragoza, Zaragoza, Spain. In Spanish (2003)

4. R. Álvarez-Nodarse. On characterizations of classical polynomials. J. Comput. Appl. Math. 196, 320-337 (2006)

5. R. Álvarez-Nodarse and J. Arvesú. On the $q$-polynomials on the exponential lattice $x(s)=c_{1} q^{s}+c_{3}$. Integral Trans. and Special Funct. 8, 299-324 (1999)

6. R. Álvarez-Nodarse, Yu. F. Smirnov, and R. S. Costas-Santos. A q-analog of the Racah polynomials and $q$-algebra $s u_{q}(2)$ in quantum optics. Journal of Russian Laser Research 27(1), 1-32 (2006)

7. G. E. Andrews. q-Series: Their Development and Application in Analysis, Number Theory, Combinatorics, Physics and Computer Algebra, volume 66 of C.B.M.S. Regional Conference Series in Math. Amer. Math. Soc., Providence, Rhode Island (1996)

8. R. Askey and S. K. Suslov. The $q$-harmonic oscillator and the Al-Salam and Carlitz polynomials. Lett. Math. Phys. 29(2), 123-132 (1993)

9. N. M. Atakishiev and S. K. Suslov. Difference analogs of the harmonic oscillator. Theoret. and Math. Phys. 85(1) , 1055-1062 (1991)

10. N. M. Atakishiev and S. K. Suslov. A realization of the $q$-harmonic oscillator. Theoret. and Math. Phys.87(1), 442-444 (1991)

11. N. M. Atakishiyev, M. Rahman, and S. K.Suslov. On classical orthogonal polynomials. Constr. Approx. 11, 181-226 (1995)

12. T. S. Chihara. An Introduction to Orthogonal Polynomials, Gordon and Breach Science Publishers, New York (1978)

13. N. J. Fine. Hypergeometric Series and Applications. Mathematical Surveys and Monographs. Amer. Math. Soc., Providence, Rhode Island (1988)

14. A. G. García, F. Marcellán, and L. Salto. A distributional study of discrete classical orthogonal polynomials. J. Comput. Appl. Math. 57, 147-162 (1995)

15. W. Hahn. Uber die Jacobischen polynome und zwei verwandte Polynomklassen. Math. Zeit. 39, 634-638 (1935)

16. R. Koekoek and R. F. Swarttouw. The Askey-scheme of hypergeometric orthogonal polynomials and its q-analogue, volume 98-17. Reports of the Faculty of Technical Mathematics and Informatics, Delft, The Netherlands (1998)

17. T. H. Koornwinder. Orthogonal polynomials in connection with quantum groups. P. Nevai (Ed.), NATO ASI Series C 294. Kluwer Acad. Publ., Dordrecht, The Netherlands, 257-292 (1990)

18. T. H. Koornwinder. Compact quantum groups and q-special functions. V. Baldoni and M.A. Picardello (Eds.), Pitman Research Notes in Mathematics Series. Longman Scientific \& Technical, New York, 257-292 (1994)

19. T. H. Koornwinder. The structure relation for Askey-Wilson polynomials. J. Comput. Appl. Math. 27, $214-226(2007)$

20. A. A. Malashin. $q$-analog of Racah polynomials on the lattice $x(s)=[s] q[s+1]_{q}$ and its connections with $6 \mathrm{j}$-symbols for the $s u_{q}(2)$ and $s u_{q}(1,1)$, quantum algebras. Master's thesis, Moscow State University, M.V. Lomonosov. In Russian (1992)

21. F. Marcellán, A. Branquinho, and J. C. Petronilho. Classical orthogonal polynomials: a functional approach. Acta Appl. Math. 34, 283-303 (1994)

22. J. C. Medem, R. Álvarez-Nodarse, and F. Marcellán. On the $q$-polynomials: a distributional study. J. Comput. Appl. Math.135, 157-196 (2001)

23. Sh. M. Nagiyev. Difference Schrödinger equation and q-oscillator model. Theoret. and Math. Phys. 102, 180-187 (1995)

24. A. F. Nikiforov, S. K. Suslov, and V. B. Uvarov. Classical Orthogonal Polynomials of a Discrete Variable. Springer Series in Computational Physics. Springer-Verlag, Berlin (1991)

25. A. F. Nikiforov and V. B. Uvarov. The Special Functions of Mathematical Physics. Birkhäuser Verlag, Basel (1988)

26. E. Routh. On some properties of certain solutions of a differential equation of the second order. Proc. London Math. Soc.16, 245-261 (1884)

27. W. Rudin. Real and Complex Analysis. McGraw-Hill, New York (1974)

28. N. Ja. Vilenkin and A. U. Klimyk. Representations of Lie Groups and Special Functions, volume I, II, III. Kluwer Academic Publishers, Dordrecht, The Netherlands (1992) 
